\title{
Can vascular endothelial growth factor C expression be of use in predicting surgical stage or prognosis in vulvar cancer?
}

\author{
Nyberg Reita $\mathrm{H}^{1, *}$, Laurila Marita², Staff Synnöve ${ }^{1,3}$ and Mäenpää Johanna $U^{1,4}$ \\ 1 Department of Gynecology and Obstetrics, Tampere University Hospital, Tampere, Finland \\ 2 Department of Pathology, Fimlab Laboratories Inc., Tampere, Finland \\ ${ }^{3}$ Laboratory of Cancer Biology, BioMediTech, University of Tampere, Tampere, Finland \\ ${ }^{4}$ Faculty of Medicine and Life Sciences, University of Tampere, Tampere, Finland
}

\begin{abstract}
Introduction: Nodal metastasis is a main prognostic factor in vulvar cancer. Increased vascular endothelial growth factor C (VEGF-C) expression has been associated with lymph node metastasis and poor prognosis in many cancers. The aim of this retrospective study was to investigate VEGF-C expression pattern in the invasive edge of vulvar cancer and in sentinel lymph node metastasis, and its association with the stage and prognosis. Methods: Tumor and sentinel lymph node samples from 44 patients were evaluated with immunohistochemistry, and the results were linked with the clinicopathological data. Results: Sixty-seven percent of primary tumors and $76 \%$ of sentinel lymph node metastases expressed VEFG-C. Positive VEGF-C expression of the primary tumor did not predict surgical Stage or sentinel lymph node involvement. The risk of relapse was not significantly higher with VEGF-C expressing tumors than with VEGF-C negative tumors (RR $2.55,95 \% \mathrm{Cl} 0.66-9.90, \mathrm{p}=0.18)$. The risk of groin recurrence was significantly lower with VEGF-C positive than negative tumors (RR 0.36 , $95 \% \mathrm{Cl} 0.16-0.79, \mathrm{p}=0.01)$. Survival was similar in both groups. No non-sentinel lymph node metastases were found in case of negative VEGF-C expression in the sentinel lymph node metastasis, whereas with positive VEGF-C expression they were found in 5/13 (38\%) of cases. Conclusions: Tumoral VEGF-C expression was not associated with higher surgical Stage or poorer prognosis in vulvar cancer. However, absence of its expression in sentinel lymph node metastasis might indicate a low risk for non-sentinel lymph node metastases.
\end{abstract}

Keywords: vulvar cancer; VEGF-C; sentinel lymph node; nodal metastasis; lymphatic spread; prognosis; recurrence

\section{Introduction}

Nodal metastatic involvement is the most important prognostic factor in vulvar cancer. Node-negative patients have a 5 -year survival rate of 70 - $98 \%$ but those with positive nodes only $12-41 \%$ [1]. Size of the primary tumor, presence of lymphovascular invasion and the depth of invasion are known to increase the risk for nodal metastasis [2-4], as well as the central location of the primary tumor [5]. Biologic prognostic variables are not as well known. Increased tumor angiogenesis and altered vessel characteristics are suggested to lead into a shorter disease-free survival [6]. Expression levels of matrix metalloproteinase-2 expression higher than $50 \%$ are also an indicator of a lower fiveyear survival rate [7]. Over-expression of tissue matrix metalloproteinases, transmembrane protein CD44 and its isoforms, thrombospondin-1, vascular endothelial growth factor (VEGF) and some G2/M pathway regulators, as well as, loss of metastasis suppressor NM23-H1 gene, seem to promote local and metastatic growth. However, not all these findings correlate with clinical prognosis $[1,8]$.

Studies in animal models and humans have shown that lymphangiogenesis in a primary tumor increases nodal metastasis [9]. Even before the metastasis actually takes place, the lymph nodes draining straight from the tumor so called sentinel lymph nodes (SLNs)- undergo remodelling processes including lymphangiogenesis, alterations in structure, lymphatic flow and immune cell composition, and increases in chemokine and cytokine production, thus creating a premetastatic niche [10]. Vascular endothelial growth factor C (VEGF-C) secreted by the primary tumor is the most important lymphangiogenic factor causing the remodelling. It alters the lymphatic vessels around the primary tumor, increases the lymphatic flow and causes expansion of lymphatic network in SLNs, all this promoting lymphatic spread [11].

*Corresponding author: Reita Nyberg, Department of Gynecology and Obstetrics, Tampere University Hospital, Tampere, Finland. Tel.: +358 50384 7500; Email: reita.nyberg@pshp.fi

Received 12 July 2017 Revised 14 August 2017 Accepted 22 August 2017 Published 31 August 2017

Citation: Nyberg RH, Laurila M, Staff S, Mäenpää JU. Can vascular endothelial growth factor $C$ expression be of use in predicting surgical stage or prognosis in vulvar cancer? J Cancer Res Ther. 2017; 5(8):50-55. DOI: $10.14312 / 2052-4994.2017-10$

Copyright: (c) 2017 Nyberg RH, et al. Published by NobleResearch Publishers. This is an open-access article distributed under the terms of the Creative Commons Attribution License, which permits unrestricted use, distribution and reproduction in any medium, provided the original author and source are credited. 
In many human cancers, over-expression of VEGF-C by primary tumor is associated with poorer prognosis. It correlates with shorter progression free survival (PFS), overall survival (OS) and lymph node metastasis in melanoma [12], and is a poor prognostic factor in nonsmall-cell lung cancer and adenocarcinoma of the lung $[13,14]$. High levels of VEGF-C expression in gastric cancer tissue imply worse overall prognosis than low VEGF-C levels [15]. Primary tumor VEGF-C expression has been reported to correlate with the possibility of lymph node metastasis in lung, oesophageal, prostate, thyroid and colorectal cancers [11].

To our knowledge, the influence of VEGF-C on the clinical course of vulvar cancer has not been studied, but one report of VEGF-C expression in 10 tissue samples has been published [16]. The aim of this study was to explore the presence of VEGF-C expression in vulvar cancer (primary tumor and SLN metastasis), and its influence on patients' surgical Stage, risk of recurrence and prognosis.

\section{Materials and methods}

\section{Patients and tissue samples}

Tissue samples from 44 vulvar cancer patients that had previously undergone vulvar surgery and a SLN mapping before complete lymph node dissection in Tampere University Hospital were used for this study. Under a 4.5-year familiarization period, a SLN mapping had been performed to all surgically treated vulvar cancer patients, and has been described elsewhere [17]. The specimens were obtained from the Tissue Biobank and Research Services FinTiB (Fimlab Laboratories Inc., Tampere, Finland). Forty-six tumor samples with representative malignant growth as well as 17 metastatic SLN samples were available for analysis.

The clinicopathological history and follow-up data of all patients were retrospectively collected from the hospital records. The history included the age at the time of the surgery, the date of the surgery, the site of the primary tumor, the type of surgery, the surgical Stage of the disease, the histopathology report, the status of the SLN (positive or negative for metastasis) and other regional lymph nodes, and whether or not other metastases were present. The follow-up data included also information of a potential adjuvant treatment and its duration, the observation date and location(s) of a recurrence, if any, the final date of the follow-up, and the date and cause of death, if it happened during the follow-up period. This data was combined with the results of the VEGF-C immunostaining for the final analysis.

\section{Immunohistochemistry}

The VEGF-C protein expression in vulvar tumors and SLN samples were evaluated by using immunohistochemistry (IHC). Representative samples from the invasive edges of the primary tumors and SLN metastases were selected for the study by an experienced pathologist (M.L.). $4 \mu \mathrm{m}$ thick sections were cut from paraffin-embedded tissue blocks using a standard microtome. For IHC staining, the slides were then deparaffinized, rehydrated, and subsequently pretreated with a PT-Module (Lab Vision, Fremont, CA) at $98^{\circ} \mathrm{C}$ for $15 \mathrm{~min}$ in $0.05 \mathrm{M}$ TrisHCl buffer, $\mathrm{pH} 9.0$ containing $0.001 \mathrm{M}$ EDTA. The primary VEGF-C antibody (Rabbit antiVEGF-C; Invitrogen, Camarillo, CA) was visualized with a PowerVision + polymer kit (Leica Biosystems Newcastle Ltd., Newcastle, UK) and diaminobenzidine as chromogen (DABImmPact, Vectorlabs, Burlingame, CA). The tissue sections were counterstained with hematoxylin (Mayer's hematoxylin, Oy FFChemicals Ab, Haukipudas, Finland). Human colon carcinoma samples, known to have a strong VEGF-C expression, were used as a positive control. Negative controls were made by omitting the primary VEGF-C antibody from the procedure.

\section{Analysis of the immunostaining}

Immunostained sections were scanned with an Aperio Scanscope XT (Aperio Technologies, Vista, CA) and visually analysed on a computer screen. Two observers (R.N. and S.S.) assessed them, blinded to the clinicopathological data of the patients. In the first round, the assessments were performed independently and the results then pooled. If the assessments of two observers were contradictory, the staining was assessed again in consensus. In all tissue samples, IHC staining intensity was scored semiquantitatively as negative (no staining at all), weak (some scattered stained cells or faint more widespread staining), moderate (more abundant widespread staining or focal intensive staining) or strong (almost all cells intensively stained). For the statistical analysis, negative and weak staining were combined as "negative" and similarly, moderate and strong staining as "positive" staining.

\section{Statistical analysis}

The concordance between two observer's assessments of the VEGF-C expression in the first evaluation round was assessed using Cohen's unweighted kappa test [18]. Associations between VEGF-C staining and clinicopathological parameters were analyzed using the Fisher's exact test, odds ratio and relative risk. Diseasespecific and progression free survival curves were calculated using Kaplan-Meier survival analysis, and compared using the log rank test. A p-value less than 0.05 was considered statistically significant. SPSS Statistics for Windows (version 19.0 released 2010, IBM Corp., Armonk, NY, USA) was used in calculation of statistical analysis.

\section{Ethical considerations}

The use of archived tissue specimens for IHC was approved by Valvira, National Supervisory Authority for Welfare and Health (6746/05.01.00.06/2009). The retrospective collection of patient data from the hospital records was approved by the Ethics Committee of Pirkanmaa Hospital District (R09066).

\section{Results}

\section{Patient and follow-up data}

The median age of patients was 76 years (range 44-93). The median follow-up time was 39 months (range 0.6 109 months). Tumor characteristics with Stage, adjuvant treatment and follow-up data are presented in Table 1.

During the follow-up, one patient died of cancer less than three weeks after surgery and two patients during the 
Table 1 Data on disease and tumor characteristics in all 44 patients.

\begin{tabular}{|c|c|c|}
\hline Variable & Definition & $\begin{array}{l}\text { Number of patients } \\
\text { (percentage of all) }\end{array}$ \\
\hline \multirow[t]{4}{*}{ FIGOa Stage } & 1 & $19(43 \%)$ \\
\hline & II & $4(9 \%)$ \\
\hline & III & $20(46 \%)$ \\
\hline & IV & $1(2 \%)$ \\
\hline \multirow{6}{*}{$\begin{array}{l}\text { Histology and Grade of the } \\
\text { primary tumor in vulva }\end{array}$} & $\mathrm{SCC}^{\mathrm{b}}$ & $43(98 \%)$ \\
\hline & Grade 1 & $22(50 \%)$ \\
\hline & Grade 2 & $14(32 \%)$ \\
\hline & Grade 3 & $7(16 \%)$ \\
\hline & $\begin{array}{l}\text { Anaplastic } \\
\text { carcinoma }\end{array}$ & $1(2 \%)$ \\
\hline & Grade 3 & $1(2 \%)$ \\
\hline \multirow[t]{3}{*}{ Sentinel node metastasis } & No & $23(52 \%)$ \\
\hline & Yes & $20(46 \%)$ \\
\hline & No SLN detected & $1(2 \%)$ \\
\hline \multirow[t]{3}{*}{$\begin{array}{l}\text { Postoperative adjuvant } \\
\text { treatment }\end{array}$} & $\begin{array}{l}\text { No adjuvant } \\
\text { treament }\end{array}$ & $22(50 \%)$ \\
\hline & $\mathrm{RT}^{\mathrm{c}}$ & $21(48 \%)$ \\
\hline & Concurrent $\mathrm{CRT}^{\mathrm{d}}$ & $1(2 \%)$ \\
\hline \multirow{2}{*}{$\begin{array}{l}\text { Alive at the end of the } \\
\text { follow-up }\end{array}$} & Yes & $22(50 \%)$ \\
\hline & No & $22(50 \%)$ \\
\hline \multirow[t]{2}{*}{ Cause of death } & $\begin{array}{l}\text { Vulvar cancer or } \\
\text { related }\end{array}$ & $15(34 \%)$ \\
\hline & Other cause & $7(16 \%)$ \\
\hline
\end{tabular}

aThe International Federation of Gynecology and Obstetrics; ${ }^{\text {bsquamous }}$

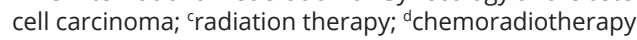

adjuvant radiotherapy before completion of the treatment (7\%, 3/44). Thirteen patients (30\%) had a recurrence after the completion of the treatment, 7 in the vulva and 6 outside the vulva. Three out of 7 patients (43\%) with vulvar recurrence were salvaged by reoperation and were alive at the end of the follow-up, while all six patients with recurrences outside vulva died of their disease. At the end of the follow-up, half of the patients were still alive.

\section{Interobserver agreement on IHC}

The interobserver agreements on the VEGF-C expression in primary tumor and SLN samples were substantial; Cohen's unweighted kappa for concordance in tumor samples was 0.69 (95\% Cl 0.48-0.90) and in SLN samples $0.72(95 \% \mathrm{Cl}$ 0.50-1.06).

\section{VEGF-C expression in primary tumors and SLN metastases}

Of 46 primary tumor samples, only 7\% (3/46) of the invasive edges of vulvar tumors did not express any VEGF-C. The expression was weak in 26\% (12/46). Thus, altogether 15 tumors (33\%) were classified as VEGF-C negative (Figure $1 a)$. The staining was moderate or strong in $53 \%(25 / 46)$ and $13 \%(6 / 46)$ of the tumor edges, respectively, and a total of 31 (67\%) tumors classified as VEGF-C positive (Figure $1 \mathrm{~b}$ ). There was no difference in median age of patients with either VEGF-C negative or VEGF-C positive tumors (75.5 vs. 76 years, $p=1.00$ ). The high- Grade tumors tended to express VEGF-C more often than the low-Grade tumors but

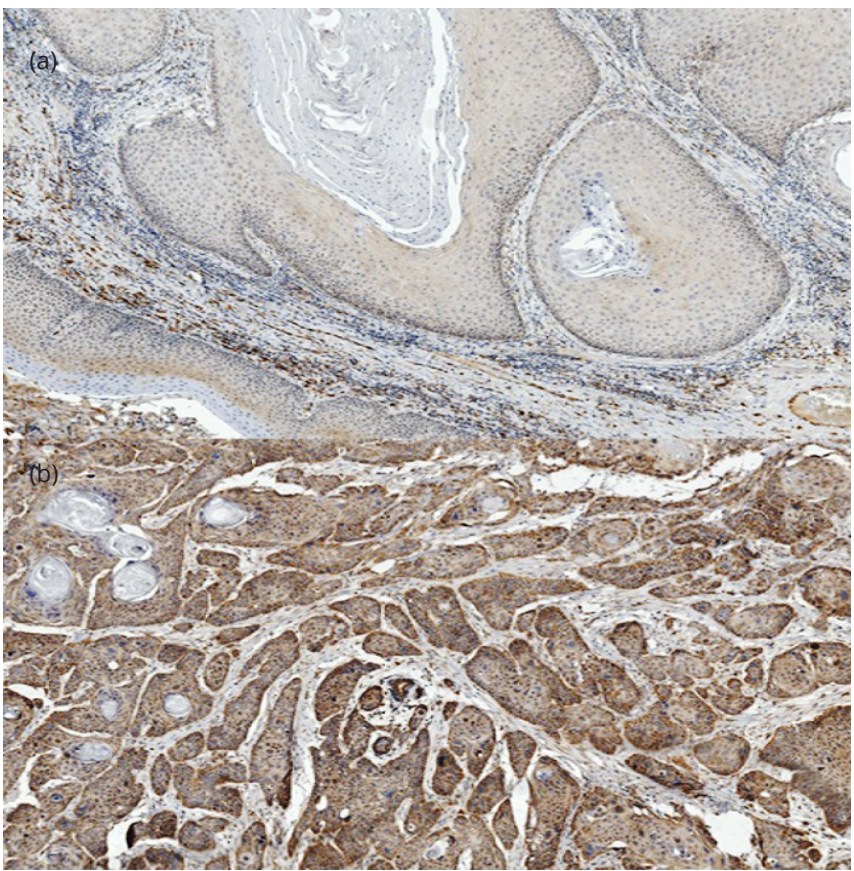

Figure 1 Examples of (a) a weak and (b) a strong VEGF-C immunostaining in squamous cell carcinoma of the vulva (magnification $\times 20$ ).

the difference was not statistically significant $(p=0.36)$, see Figure 2. For one tumor, the Grade was not available.

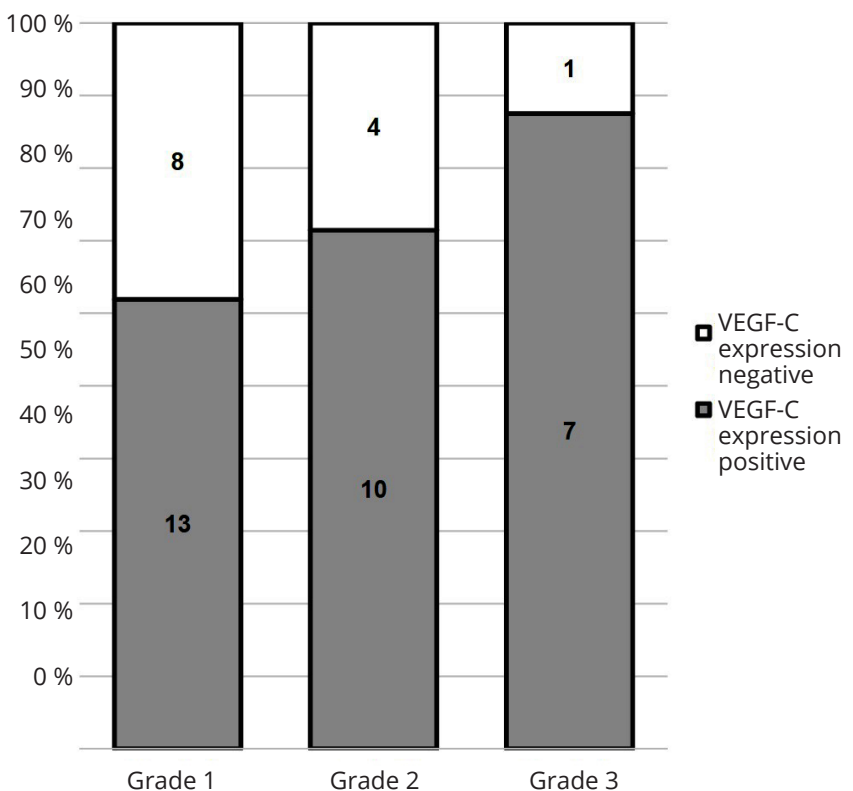

Figure 2 VEGF-C expression in invasive edges of vulvar cancer according to the histological Grade.

\section{Tumoral VEGF-C expression and surgical Stage}

At the time of the surgery, 17 out of 30 (57\%) VEGF-C positive and 8 out of 14 (57\%) VEGF-C negative tumors were advanced (> FIGO Stage I). The risk for more advanced surgical Stage was the same with VEGF-C positive and negative tumor groups (OR 0.98, 95\% Cl 0.27-3.53, $\mathrm{p}=$ 0.98). Also, the risk of having SLN metastasis at the time of surgery did not significantly differ between VEGF-C positive or negative tumors $(47 \%, 14 / 30$ and $46 \%, 6 / 13$, respectively; OR $1.02,95 \% \mathrm{Cl} 0.28-3.77, \mathrm{p}=0.98$ ). 


\section{VEGF-C expression in SLN metastasis}

The SLN metastases were VEGF-C negative in $24 \%(4 / 17)$ and VEGF-C positive in $76 \%$ (13/17) of the cases (Figure 3). When the primary tumor was VEGF-C positive, the SLN metastasis expressed VEGF-C in $91 \%(10 / 11)$ of the cases as compared to $50 \%(3 / 6)$ of the SLN metastases in the VEGF-C negative vulvar tumors, but the difference did not reach a statistical significance $(p=0.099)$.

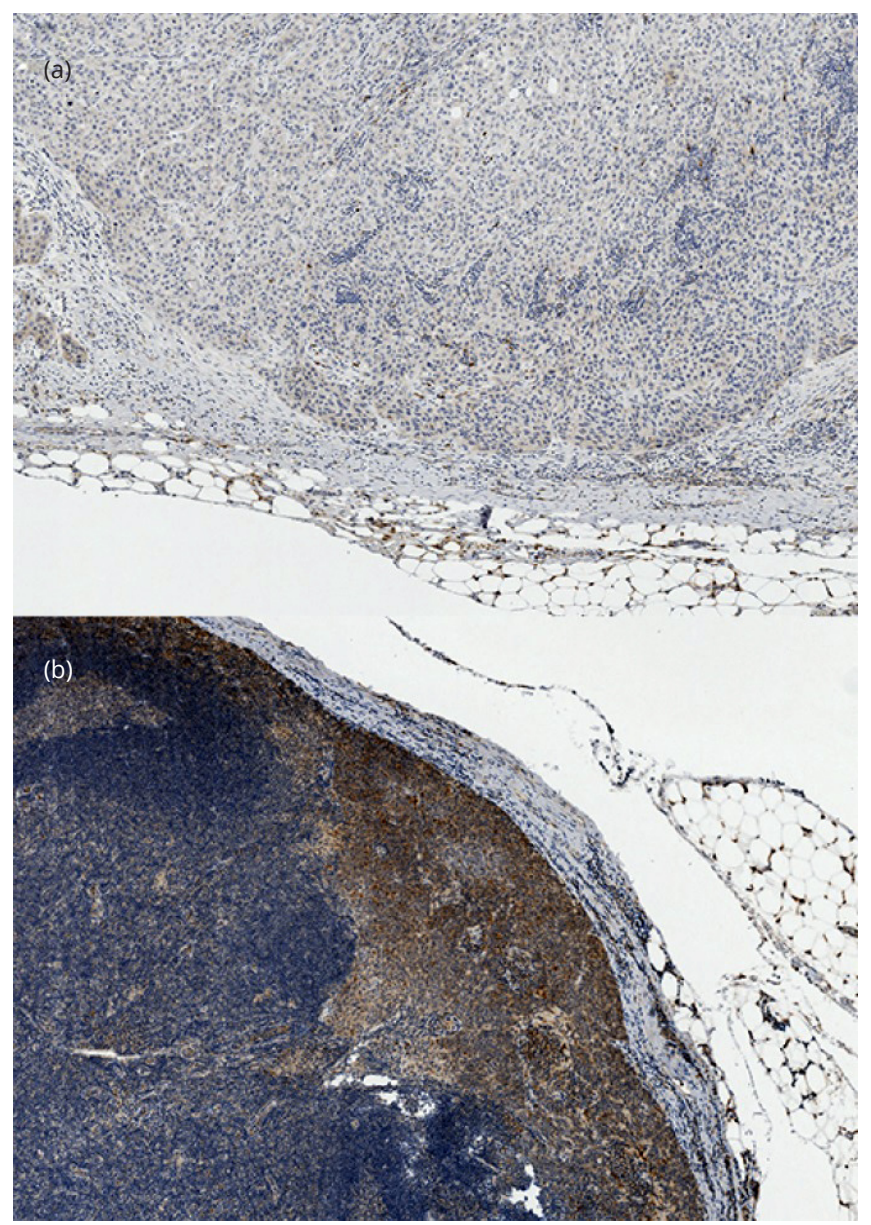

Figure 3 Examples of (a) a weak and (b) a strong VEGF-C immunostaining in sentinel lymph node metastases (magnification $\times 20$ ).

When the SLN metastasis expressed VEGF-C, in 5 cases out of $13(38 \%)$ metastatic non-SLNs were also found. However, in four cases when the SLN metastasis was VEGF-C negative, no other LN metastases were found $(0 / 4$; OR $5.82,95 \% \mathrm{Cl}$ $0.26-130.89, p=0.267$ ). The positive predictive value of VEGF-C expression in the SLN metastasis in relation to the non-SLN metastases was $38 \%$ and the negative predictive value $100 \%$, bearing in mind the small number of VEGF-C negative SLN metastases.

\section{VEGF-C expression and the clinical course of the disease}

In primary tumors: Excluding three patients that died before the completion of the primary treatment, the patients with VEGF-C positive primary tumors seemed to relapse more often $(39 \%, 11 / 28)$ during the follow-up than the patients with VEGF-C negative tumors (15\%, 2/13), although the risk was not statistically significant (RR $2.55,95 \%$ Cl 0.66-9.90, p $=0.18)$. The VEGF-C positive tumors recurred mostly in the vulvar area $(64 \%, 7 / 11)$ while the VEGF-C negative tumors recurred in the inguinal area $(100 \%, 2 / 2)$. The risk of groin recurrence was significantly lower, when the tumor expressed VEGF-C (RR 0.36, 95\% CI 0.16-0.79, p = 0.01). The disease-specific survival (DSS) as a function of the VEGF-C expression of the primary tumors is shown in Figure 4. No difference was observed (Log rank test, $p=$ 0.83 ). There seemed to be a trend towards a better PFS in patients with VEGF-C negative tumors when compared to VEGF-C positive tumors, see Figure 5 . However, this trend did not reach a statistical significance (Log rank test, $p=$ 0.19).

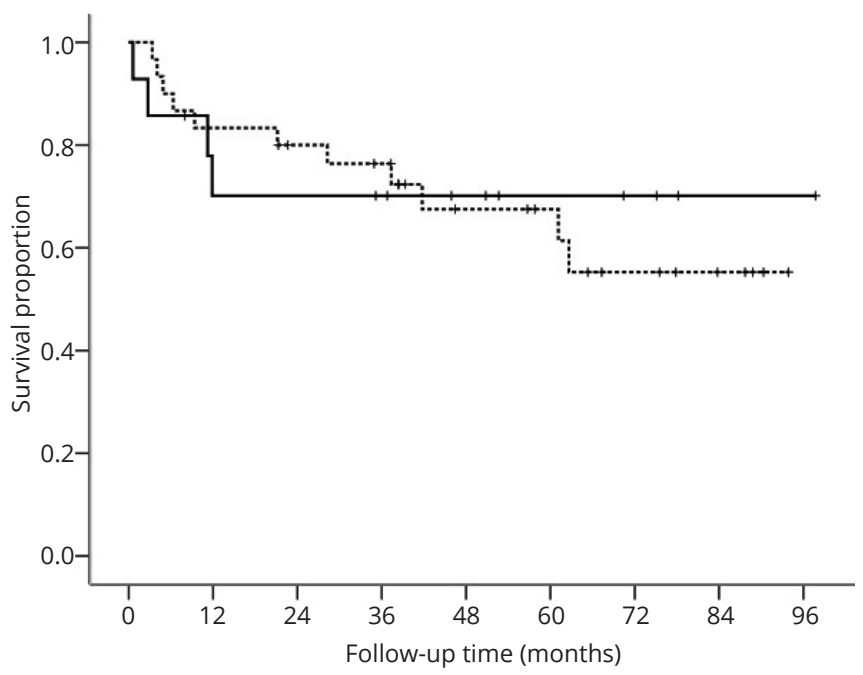

Figure 4 Disease-specific survival analysis according to VEGF-C expression of vulvar tumors (log rank test $\mathrm{p}=0.83$ ).

Footnote: - negative VEGF-C expression; --- positive VEGF-C expression; + censored

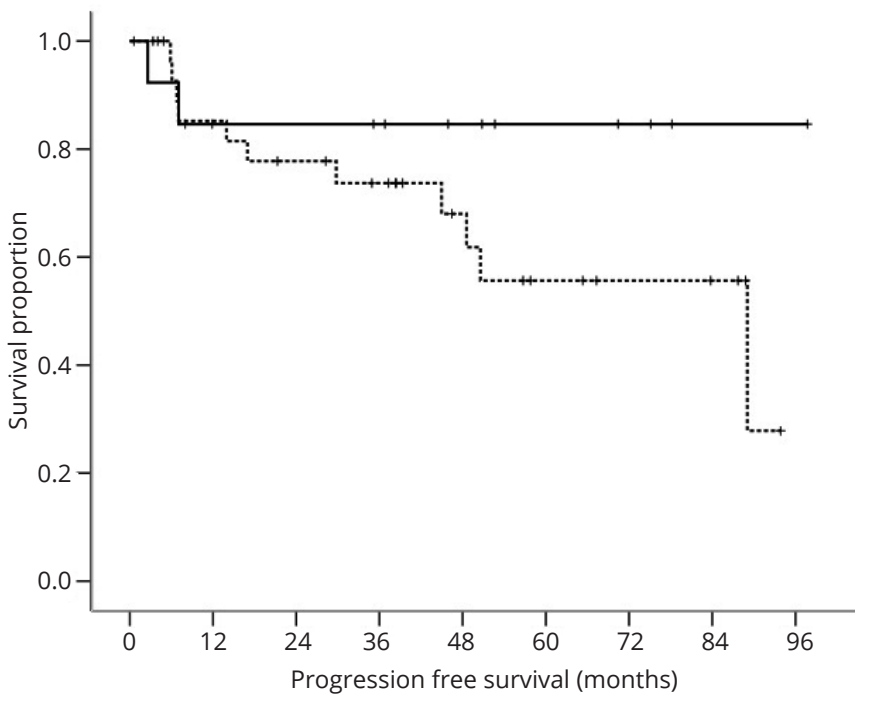

Figure 5 Progression-free survival analysis according to VEGF-C expression of vulvar tumors (log rank test $\mathrm{p}=0.19$ ).

Footnote: - negative VEGF-C expression; --- positive VEGF-C expression; + censored

In SLN metastases: There was no difference in the risk of recurrence between patients with VEGF-C positive and negative SLN metastases $(5 / 12$ and $1 / 3$, respectively, RR 1.25, $95 \% \mathrm{Cl} 0.22-7.08, \mathrm{p}=0.80$ ), respectively. The only recurrence in the group with VEGF-C negative SLN metastasis appeared in vulvar area whereas three out of five recurrences $(60 \%)$ in the group with VEGF-C positive SLN metastasis appeared in inguinal area and two 
recurrences (40\%) in vulvar area. These groups were too small for statistical analysis.

\section{Discussion}

According to the results of this study, the primary tumors of most vulvar cancers express VEGF-C on their invasive edges. The frequency of expression tended to correlate positively with histological Grade, but the difference did not reach statistical significance. VEGF-C was also expressed in three quarters of the SLN metastases, more often when the primary tumor expressed it, although the difference was again not significant. VEGF-C expression of the primary tumor was not associated with higher surgical Stage or risk of nodal metastasis, nor did it have any statistically significant impact on DSS or PFS. A negative VEGF-C expression in a SLN metastasis could be a favourable indicator of cancer-free non-SLNs.

When considering the rarity of vulvar cancer, our sample size of 44 tumors was representative. However, it was still not large enough to show statistical significance between different groups even when a trend was observed. The strengths of this study were a long follow-up time and its clinical orientation. Our median follow-up of 39 months was long enough for relapses to become evident. We chose to keep the IHC scoring simple, bearing in mind its potential application to clinical patient care.

Our finding of the frequency of VEGF-C expression in vulvar cancer differs from the only other published report by Jach et al. In their much smaller population, VEGF-C expression was observed only in 10\% (1 out of 10) of vulvar squamous cell cancer (SCC) cases. The carcinoma specimens they used for the IHC analysis were individually selected [16]. However, the authors did not specify, which part of the tumor these specimens represented nor did they tell the histological Grades of vulvar tumors - a feature that in our study seemed to effect on the VEGF-C expression. When the expression of VEGF-C was studied from 111 cervical SCC samples by Gombos et al., it was found to be heterogeneous within the tumors. The expression was significantly higher in the marginal portions of carcinomas compared with the central regions [19]. We also focused on the invasive edge of vulvar tumors and noticed the same phenomenon as Gombos et al. Furthermore; the semiquantitative scoring system Jach et al. used to assess VEGF-C expression took into account the percentage of VEGF-C positive cells. If central parts of the vulvar tumors were used for the immunostaining analysis, it might have diminished their scores even when the staining was strong.

According to literature, SCCs in many different organs express VEGF-C, i.e., tumors of oral cavity [20], oesophageal cancer [21] and cervical cancer [22]. The positive expression has been associated with poorer prognosis and higher risk of lymphatic metastasis. However, in our study tumoral VEGF-C expression did not predict surgical Stage or frequency of the SLN involvement.

We observed that VEGF-C positive cancers tended to recur more often than VEGF-C negative cancers, and therefore also PFS seemed to be more favorable in patients with VEGF-C negative tumors, albeit not significantly. In our study population, $43 \%$ of patients with a vulvar recurrence were successfully salvaged, while all groin recurrences were fatal. The risk of groin recurrence was significantly lower in VEGF-C positive tumor group than in VEGF-C negative tumor group. Better prognosis of the local recurrences compared to the groin recurrences might partly explain why the positive tumoral VEGF-C expression had no impact on the disease-specific survival. The VEGF-C expression in SLN metastasis was not associated with the recurrence rate.

At the moment, the only known prognostic factor for non-SLN metastasis in vulvar cancer in relation to characteristics of a SLN metastasis is the size of the metastasis. GROINSS-V study showed that the risk for nonSLN metastasis increases with the size of SLN metastasis. No size cut-off existed below which chances of non-SLN metastases would be close to zero. Therefore, additional treatment was recommended to all SLN positive patients, but it always increases side effects and lowers quality of life [23]. Our study suggests that a negative VEGF-C expression in SLN metastases could act as an indicator of cancer-free non-SLNs. However, the small number of metastatic SLN samples (four) limits drawing conclusions and this finding should be tested in a larger population. If reproducible, VEGF-C expression in SLN metastasis could serve as another prognostic factor when considering additional treatment.

\section{Conclusion}

VEGF-C expression was frequent in the invasive edges of malignant vulvar tumors and their SLN metastases. If verified in a larger population, the lack of VEGF expression in SLN metastasis may in the future prove to be a useful indicator of lower risk for non-sentinel lymph node metastasis. Otherwise, VEGF-C expression in primary tumors did not seem to function as a helpful indicator of surgical Stage or prognosis in vulvar cancer.

\section{Acknowledgements}

The authors want to thank Prof. Jorma Isola, MD, PhD, for his valuable advice and consultation in relation to IHC, Heini Huhtala, M.Sc. for her assistance with the statistical analysis and AV consultant Mika Martikainen for his help with the digital artwork.

\section{Funding}

This study has been supported by Research Grants from Pirkanmaa Hospital District's Science Centre (Grant number 9H183), Competitive Research Funding of Tampere University Hospital (Grant number 9L062) and Research Foundation of Obstetrics and Gynecology, Finland (Personal Grant for R.N.).

\section{Conflicts of interest}

Authors declare no other conflicts of interest than the abovementioned Grants (J.M., R.N.). 


\section{References}

[1] Gadducci A, Tana R, Barsotti C, Guerrieri ME, Genazzani AR. Clinicopathological and biological prognostic variables in squamous cell carcinoma of the vulva. Crit Rev Oncol Hematol. 2012; 83(1):71-83.

[2] Ayhan A, Velipasaoglu M, Salman MC, Guven S, Gultekin M, et al. Prognostic factors for recurrence and survival in primary vulvar squamous cell cancer. Acta Obstet Gynecol Scand. 2008; 87(11):11431149.

[3] Woelber L, Eulenburg C, Choschzick M, Kruell A, Petersen C, et al. Prognostic role of lymph node metastases in vulvar cancer and implications for adjuvant treatment. Int J Gynecol Cancer. 2012; 22(3):503-508.

[4] Sznurkowski J], Milczek T, Emerich J. Prognostic factors and a value of 2009 FIGO staging system in vulvar cancer. Arch Gynecol Obs. 2013; 287(6):1211-1218.

[5] Hinten F, van den Einden LC, Cissen M, IntHout J, Massuger LF, et al. Clitoral involvement of squamous cell carcinoma of the vulva: localization with the worst prognosis. Eur J Surg Oncol. 2015; 41(4):592598.

[6] Näyhä V V , Stenbäck FG. Increased angiogenesis is associated with poor prognosis of squamous cell carcinoma of the vulva. Acta Obstet Gynecol Scand. 2007; 86(11):1392-1397.

[7] Zanvettor PH, Filho DF, Soares FA, Neves AR, Palmeira LO. Study of biomolecular and clinical prognostic factors in patients with cancer of the vulva undergoing surgical treatment. Int J Gynecol Cancer. 2014; 24(4):766-772.

[8] Wang Z, Slipicevic A, Førsund M, Trope CG, Nesland JM, et al. Expression of CDK1Tyr15, pCDK1Thr161, Cyclin B1 (Total) and pCyclin B1Ser126 in vulvar squamous cell carcinoma and their relations with clinicopatological features and prognosis. PLoS One. 2015; 10(4):e0121398.

[9] Podgrabinska S, Skobe M. Role of lymphatic vasculature in regional and distant metastases. Microvasc Res. 2014; 95:46-52.

[10] Pereira ER, Jones D, Jung K, Padera TP. The lymph node microenvironment and its role in the progression of metastatic cancer. Semin Cell Dev Biol. 2015; 38:98-105.

[11] Stacker SA, Williams SP, Karnezis T, Shayan R, Fox SB, et al. Lymphangiogenesis and lymphatic vessel remodelling in cancer. Nat Rev Cancer. 2014; 14(3):159-172.

[12] Boone B, Blokx W, De Bacquer D, Lambert J, Ruiter D, et al. The role of VEGF-C staining in predicting regional metastasis in melanoma. Virchows Arch. 2008; 453(3):257-265.

[13] Kilvaer TK, Paulsen EE, Hald SM, Wilsgaard T, Bremnes RM, et al. Lymphangiogenic markers and their impact on nodal metastasis and survival in non-small cell lung cancer - A structured review with metaanalysis. PLoS One. 2015; 10(8):e0132481.

[14] Kojima H, Shijubo N, Yamada G, Ichimiya S, Abe S, et al. Clinical significance of vascular endothelial growth factor-C and vascular endothelial growth factor receptor 3 in patients with T1 lung adenocarcinoma. Cancer. 2005; 104(8):1668-1677.

[15] Cao W, Fan R, Yang W, Wu Y. VEGF-C expression is associated with the poor survival in gastric cancer tissue. Tumour Biol. 2014; 35(4):33773383.

[16] Jach R, Dyduch G, Radon-Pokracka M, Przybylska P, Mika M, et al. Expression of vascular endothelial growth factors VEGF-C and -D, VEGFR-3, and comparison of lymphatic vessels density labeled with D2-40 antibodies as a prognostic factors in vulvar epithelial neoplasia (VIN) and invasive vulvar cancer. Neuro Endocrinol Lett. 2011; 32(4):530-539.

[17] Nyberg RH, livonen M, Parkkinen J, Kuoppala T, Mäenpää JU. Sentinel node and vulvar cancer: A series of 47 patients. Acta Obstet Gynecol Scand. 2007; 86(5):615-619.

[18] Cohen J. A coefficient of agreement for nominal scales. Educ Psychol Meas. 1960; 20(1):37-46

[19] Gombos Z, Xu X, Chu CS, Zhang PJ, Acs G. Peritumoral lymphatic vessel density and vascular endothelial growth factor $C$ expression in earlystage squamous cell carcinoma of the uterine cervix. Clin Cancer Res. 2005; 11(23):8364-8371.

[20] Karatzanis AD, Koudounarakis E, Papadakis I, Velegrakis G. Molecular pathways of lymphangiogenesis and lymph node metastasis in head and neck cancer. Eur Arch Otorhinolaryngol. 2012; 269(3):731-737.

[21] Peng J, Shao N, Peng H, Chen LQ. Prognostic significance of vascular endothelial growth factor expression in esophageal carcinoma: A meta-analysis. J Balk Union Oncol. 2013; 18(2):398-406.
[22] Zhang J, Liu J, Zhu C, He J, Chen J, et al. Prognostic role of vascular endothelial growth factor in cervical cancer: A meta-analysis. Oncotarget. 2017; 8(15):24797-24803.

[23] Oonk MH, van Hemel BM, Hollema $H$, de Hullu JA, Ansink AC, et al. Size of sentinel-node metastasis and chances of non-sentinel-node involvement and survival in early stage vulvar cancer: Results from GROINSS-V, a multicentre observational study. Lancet Oncol. 2010; 11(7):646-652. 